\title{
Craniofacial morphology is distinctive in parents of children with orofacial clefting
}

\section{Are there characteristic features of the craniofacial skeleton in parents of children who have orofacial clefting (OFC)?}

\author{
McIntyre GT, Mossey PA. The craniofacial morphology of the \\ parents of children with orofacial clefting: a systematic review of \\ cephalometric studies. J Orthodont 2002; 29:23-29
}

Data sources Cochrane Library, Medline, HealthStar, POPLINE, SDLINE, SPACELINE, EMbase, Old Medline, CINHAL, ASKSAM, the orthodontic reference database (1950-1997), European Clearing House on Health Systems reform, UK National Research Register and hand searching of Cleft Palate-Craniofacial Journal and bibliographies of retrieved publications.

Study selection The search strategy was based on the key words, "parent", "cephalometry" and "'cleft". Studies written in any language were included but case-reports and case series were excluded. Data extraction and synthesis A range of clinical parameters were extracted from the studies with statistical analysis when available. Studies were assessed for quality but methodological variation and insufficient consistency in study design precluded synthesis of data.

Results Parental craniofacial morphology in OFC is distinctive.

Conclusions The parental craniofacial complex in OFC is distinctive in comparison with parents of children without cleft lip and palate but there is insufficient information to localise these differences. The quality of available data is limited.

\section{Commentary}

Intrinsic variations in craniofacial form may be significant in the early diagnosis, counselling and prevention of OFC. This review addresses the challenge of identification, assessment and characterisation of craniofacial morphology of parents of children who have OFC. They also assess correlation of craniofacial morphology in cleft lip and palate and the differences, if any, therein.

The authors infer that because craniofacial form of individuals with OFC is distinctive, hereditary factors influence that craniofacial form. Therefore, the craniofacial form of the biological parents of OFC children could also be distinctive. The presuppositions of the authors in terms of the need to identify parental craniofacial form and relate it to aetiopathogenesis of OFC tend to overshadow the clear objectives they have defined in the research questions.

The comprehensive search strategy used will have identified most if not all the relevant studies, of which only 15 met all the criteria. The authors have correctly observed that the in 15 retrospective case-control observational studies, it would be more appropriate to use the term 'comparison group' rather than control.

It is evident that the authors have been hampered by both the quality as well as the methodology of the included studies. To arrive at some logical conclusions and answer the key questions raised at the beginning of the study a synthesis of data would be essential. Data abstraction defines a direction but does not provide conclusive evidence.

Statistically significant variables from univariate statistics were evaluated for clinical significance using well-defined criteria and the data abstracted were presented in unweighted form. Multivariate analysis data could not be synthesised. The description of the efforts of authors to analyse data from various studies makes interesting reading. The use of a cluster analysis to produce a series of male and female values above or below which a potential parent could be classified as 'at risk' is perhaps the type of answer that would be sought from a review. There is also a wide variation in the outcome of discriminant analysis in various studies.

Some directions and trends do emerge. The review finds that parental craniofacial morphology is distinct compared to the population as a whole. There is insufficient evidence to either characterise the craniofacial morphology or to correlate the differences between the Cleft Lip and Palate and Cleft Palate groups. The inadequacy of conventional cephalometric analysis to assess shape is well established. Sexual dimorphism and ethnic and geographical variability are responsible for the conflicting results in various studies. This would limit the application of the results to a local population group.

Identifying patients who have extreme craniofacial anomalies such as OFC is a challenge. Would it be possible to conclude from a cephalometric examination of potential parents whether the offspring would be at risk for Cleft Lip/Cleft Palate? A meta-analysis may have yielded answers. There is, however, a need to augment conventional cephalometric with morphometric tools to provide conclusive evidence, as suggested by the authors.

The authors are to be complimented on choosing to focus on a significant issue and providing a comprehensive review of the cephalometric studies that are available. The inadequacy of suitable evidence has dogged orthodontics. Highlighting the shortcomings may help to show the directions needed in future research. After all, it is difficulty not facility that brings out the best in man. Why should research be any different?

\section{Anmol S Kalha}

Department of Orthodontics, Director and Coordinator, Centre for Evidence Based Dentistry, Davangere, India

Evidence-Based Dentistry (2003) 4, 16.

doi:10.1038/sj.ebd.6400167 